\title{
An Anatomical Model of the Knee Joint Obtained by Computer Dissection
}

\author{
S. Martelli ${ }^{1}$, F. Acquaroli ${ }^{1}$, V. Pinskerova ${ }^{2}$, A. Spettol $^{1}$, and A. Visani ${ }^{1}$ \\ ${ }^{1}$ Istituti Ortopedici Rizzoli, Lab. Biomechanics, Bologna, Italy \\ ${ }^{2}$ Charles University, Prague, Check Republic \\ s.martelli@biomec.ior.it
}

\begin{abstract}
This paper reports the analysis of the articular surfaces of the femur and the tibia in normal knees. Six cadaveric joints were digitized with FARO Arm electrogoniometer and elaborated off-line fitting the profiles of multiplanar sections with least square curves. We found that: the femoral medial condyle can be represented by an ellipsoid with its main axis in the AP direction, semi-axes equal to 30 and $23 \pm 2 \mathrm{~mm}$ (roughly spherical posteriorly) and circular ML section (radius $=20 \pm 2 \mathrm{~mm}$ ); the tibial medial plateau can be approximated by a semi-cylinder with its main axis in the AP direction and circular ML section (radius $=22 \pm 1.3 \mathrm{~mm}$ ); the femoral lateral condyle can be represented by a ellipsoid with semi-axes equal to 26 and $20 \pm 4 \mathrm{~mm}$ and an elliptic ML section with a sloped major axis parallel to the tibial spine; the articulating tibial surface in the lateral compartment is approximately flat.
\end{abstract}

Keywords: femur, tibia, knee, anatomy, electrogoniometer.

\section{Introduction}

Computer techniques and new electronic equipment are progressively changing medical investigations and treatment, but have been seldom used for accurate anatomical investigations [1], [2], [3]. In particular anatomical studies of the knee could benefit from an improved accurate knowledge of its structures, as still some controversial opinions exist on the geometry of the knee joint and poor quantitative information is available in $3 \mathrm{D}$.

The femoral condyles in the sagittal plane have been described as spirals [4], [5], [6] [7] or circles, at least posteriorly, [4], [5], [8], [9], [10], [11], [12], [13], [14], [15].

The description of the femoral shape in the frontal plane and in $3 \mathrm{D}$ is scarcer. To the authors' knowledge the frontal sections of the femur were measured only by Wismans [16] and by Kurosawa [8] in 3D studies, and the surfaces were fitted respectively with a spline of degree 4 or a sphere.

The description of tibial anatomy in the literature is almost always qualitative and anyway very poor [17], [6], [11], [18], [19]. It was approximated to one or more planes in [15], [9] , [6] [4], or a complex parametric surface in [16],[20]. 
In this paper we report an original anatomical study of six human specimens based on the use of an accurate digitizer and in off-line computer elaboration of data, least square fitting of sections and statistical evaluation. The goal of this study is to update the classical anatomical data, in order to complement present knowledge and provide reliable numerical data for 3D modeling and computer-assisted reconstruction. This work integrates a previous work by two of the authors using a different numerical approach [21].

\section{Material and Methods}

Six normal knee specimens were digitized using the FARO Arm electrogoniometer (FARO Technologies, Lake Mary, FL, USA; $0.3 \mathrm{~mm} / 0.3^{\circ}$ accuracy), obtaining 6634 points on average on the femur and 3335 points on average on the tibia. For display purposes the configuration of the joint at extension, $10^{\circ}, 30^{\circ}, 60^{\circ}, 90^{\circ}, 120^{\circ}$ of flexion was recorded via pins implanted in the bones. An anatomical joint reference frame was established according to the clinical conventions, choosing the vertical axis along the tibial anatomical axis and the perpendicular anterior-posterior direction. The knee anatomy was studied in the 3D computer reconstruction and 2D sections in different orientations. In particular this study consists in a statistical evaluation of the shape of femoral and tibial articulating surfaces in $2 \mathrm{~mm}$ consecutive sagittal (30 per knee on average), frontal (17 per knee on average) and coronal sections ( 9 per knee). The 2D profile of the articulating area was fitted by least square circles, ellipses and line to find the best approximation (Figure 1).


Fig. 1. Software elaboration of knee data: 3D reconstruction (left) and sagittal sections (right) 


\section{Results}

\subsection{Femoral Shape in Sagittal Section}

In the $15 \mathrm{~mm}$ central part of the medial condyle the whole profile was fitted (residual $<0.3 \mathrm{~mm}$ ) by an ellipse with mean $\mathrm{e}=0.62 \pm 0.08$, and semi-axes $\mathrm{R} 1=30.4 \pm 1.9$ $\mathrm{mm}, \mathrm{R} 2=23.18 \pm 1.9 \mathrm{~mm}$. The ellipse fitted also the whole patello-femoral joint anteriorly in all knees and posteriorly an arc often going beyond the contact point between tibia and femur at $120^{\circ}$ (but not including the final 5-10 mm arc), except in a few sections of three knees. Table 1 reports the mean eccentricity and axes of the least-square ellipses fitting in each knee parallel sagittal profiles in a range of equivalent variable arcs and sections residuals $<0.4 \mathrm{~mm}$.

The sagittal profile of this part of the medial condyle could also be interpolated by two different circles within the acquisition accuracy (i.e. residuals $<0.4 \mathrm{~mm}$ ), one posterior and one fitting a smaller anterior arc. The identification of posterior circle was quite stable, from a numerical point of view, so that the variability of possible circles fitting arcs $>60^{\circ}$ with residuals $<0.4 \mathrm{~mm}$ was limited in each section and also in adjacent sections. However the least square fitting of the remaining anterior part of the tibio-femoral joint was numerically uncertain, as it was affected by a very high variability due to the small size of the fitted arc (usually $<40^{\circ}$ ) and significant individual differences (e.g. knee 1 and 2). Details of this fitting and the discussion of its implications are reported in a different work [21].

In the $13 \mathrm{~mm}$ central part of the lateral condyle, and around the section of maximal curvature, the articulating profile could be fitted by an ellipse with mean e $=0.48 \pm$ 0.05 , and semi-axes R1 $=26.4 \pm 3.5 \mathrm{~mm}, \mathrm{R} 2=20.4 \pm 4.4 \mathrm{~mm}$ (Table 2). The ellipse fitted a large arc, extending from the posterior contact in full flexion to a large part of the patello - femoral joint, except in one knee in which the end of the tibio-femoral joint was clearly marked by an evident wedge. In 2 knees and single sections of the other specimens the lateral profile clearly showed a similar "dent", even if smaller and under the fitting resolution.

The sagittal profile of this part of the lateral condyle could also be interpolated by circles. Being more "round" (lower mean eccentricity) than the medial compartment, the least square fitting of almost the whole profile was possible with a single circle and the small remaining part could be described by as a line in one knee or as an arc of a larger circle, which was numerically undefined (the mean arc was $30^{\circ}$ ). [21]

\subsection{Tibial Shape in Sagittal Section}

The central concavity of the medial plateau could be very clearly fitted by an arc of a circle in 2 knees, it could be fitted equivalently by an arc of circle or two lines making a large angle in most knees degenerating into a single flat line in several sections [21]. The circle fitting the medial sagittal tibial plateau in this central articular region had a mean radius $\mathrm{R}=75 \pm 18 \mathrm{~mm}$ (mean on sections where this fitting can be computed). However, the small size of the tibial sagittal profile made the numerical interpolation more unstable than in the femoral sagittal sections (higher standard deviation 
in the same knee) and less reliable (e.g. even in the same section each data have more than $5 \mathrm{~mm}$ error on $\mathrm{R}$ for $35^{\circ}$ arc).

The shape of the lateral plateau in sagittal sections was quite regular, because it basically stayed convex in all sections, gradually changing the sharp edge of the tibial eminence to a circular convex profile with radius $36.02 \pm 11.3 \mathrm{~mm}$, over an arc of $69.44^{\circ} \pm 18.1^{\circ}$.

Table 1. Fitting of the medial femoral sagittal profile with an ellipse: mean eccentricity, major semi-axis (R1) and minor semi-axis (R2) \pm standard deviation of the fitting in parallel sections

\begin{tabular}{cccc}
\hline Knee & Eccentricity & $\mathrm{R} 1(\mathrm{~mm})$ & $\mathrm{R} 2(\mathrm{~mm})$ \\
\hline 1 & $0.68 \pm 0.08$ & $31.3 \pm 3.0$ & $22.4 \pm 0.7$ \\
2 & $0.67 \pm 0.02$ & $30.3 \pm 0.6$ & $22.4 \pm 0.6$ \\
3 & $0.59 \pm 0.06$ & $33.8 \pm 1.0$ & $27.2 \pm 1.0$ \\
4 & $0.54 \pm 0.05$ & $27.9 \pm 1.9$ & $23.3 \pm 1.2$ \\
5 & $0.64 \pm 0.03$ & $28.6 \pm 1.8$ & $21.9 \pm 0.8$ \\
6 & $0.62 \pm 0.04$ & $30.4 \pm 2.0$ & $21.9 \pm 1.9$ \\
\hline Mean & $0.62 \pm 0.05$ & $30.4 \pm 1.9$ & $23.2 \pm 2.0$ \\
\hline
\end{tabular}

Table 2. Fitting of the lateral femoral sagittal profile with an ellipse: mean eccentricity, major semi-axis (R1) and minor semi-axis (R2) \pm standard deviation of the fitting in parallel sections

\begin{tabular}{cccc}
\hline Knee & Eccentricity & $\mathrm{R} 1(\mathrm{~mm})$ & $\mathrm{R} 2(\mathrm{~mm})$ \\
\hline 1 & $0.58 \pm 0.02$ & $27.3 \pm 1.2$ & $22.6 \pm 1.5$ \\
2 & $0.57 \pm 0.03$ & $26.3 \pm 2.3$ & $21.4 \pm 2.0$ \\
3 & $0.57 \pm 0.03$ & $30.1 \pm 0.9$ & $25.1 \pm 0.5$ \\
4 & $0.65 \pm 0.07$ & $31.6 \pm 3.8$ & $13.5 \pm 1.17$ \\
5 & $0.52 \pm 0.02$ & $22.7 \pm 1.4$ & $19.4 \pm 1.5$ \\
6 & $0.0 \pm 0.02$ & $20.5 \pm 0.8$ & $20.5 \pm 0.8$ \\
\hline Mean & $0.48 \pm 0.05$ & $26.4 \pm 3.5$ & $20.4 \pm 4.4$ \\
\hline
\end{tabular}

\subsection{Femoral Shape in Frontal Section}

The articular surface of the medial condyle appeared circular, or occasionally elliptic with a low eccentricity. A circle with average radius $\mathrm{R}=20.3 \pm 2.4 \mathrm{~mm}$ fitted the whole condyle in all knees.

The lateral condyle appeared almost flat near the contact with the tibial spine, and could be fitted by an ellipse with $\mathrm{e}=0.81 \pm 0.07, \mathrm{R} 1=16.5 \pm 2.12 \mathrm{~mm}, \mathrm{R} 2=9.3 \pm$ $1.3 \mathrm{~mm}$ and the longer axis parallel to the lateral wall of the tibial spine, making an average $-17.1^{\circ} \pm 2.5^{\circ}$ with our horizontal plane. The part of the profile in contact with the tibia and near the notch could be fitted also by a line with similar slope, and in one knee individual deformation of the bone or cartilage made this fitting clearly better than the elliptic interpolation, although numerically unstable [21].

The posterior frontal sections of the femoral condyles (in the extension position) became more and more circular in both compartments. In the posterior part of the femur the frontal sections of both compartments became very similar, profiles could be well fitted by circles with decreasing radius (maximum $\mathrm{R}=19.4 \pm 1.1$ ). 


\subsection{Tibial Shape in Frontal Section}

In the central part of the tibial plateau the frontal profile was very typical, clearly circular in all knees and conform to the femoral profile (average radius $=21.9 \pm 1.3 \mathrm{~mm}$ over an arc of $69.2^{\circ} \pm 14^{\circ}$, including tibial the medial side of the spine and excluding the curved medial extremity).

The lateral tibial plateau was more "flat" than the medial one, with small anterior and posterior flat portion, with a wedge between the tibial spine and the flat plateau. The frontal profile was fitted by 2 lines making an average angle of $167^{\circ}$. The line fitting the lateral wall of the tibial plateau (average slope $-21.3^{\circ} \pm 3.8^{\circ}$ ) was roughly parallel to the ellipse fitting the femoral lateral condyle or the line describing its medial portion .

\subsection{Joint Shape in Coronal Section}

The profile of the femoral condyles varied as the coronal sections moved proximally. Both medial and lateral condyles appeared "round" posteriorly, with increasing radius becoming maximal and stable in the area corresponding roughly to the contact area between tibia and femur at $90^{\circ}$. In this region (around $25 \mathrm{~mm}$ over the tibial plateau \pm $4 \mathrm{~mm}$ ) selective coronal sections were examined to describe the posterior curvature of the femoral condyles. The profile was well fitted by a circle in both compartments, but the lateral side was bigger $(19.8 \pm 2.5 \mathrm{~mm}$ average radius) than the medial one (17.3 $\pm 1.8 \mathrm{~mm}$ average radius)

The coronal sections of the tibial plateau did not provide consistent profiles, because of the almost horizontal distribution of data. However, the coronal sections of the tibial spine showed a repeatable interesting pattern in the articular region of the two compartments, height $4 \mathrm{~mm}$ on average. The medial compartment had a linear profile, while the lateral one was $\mathrm{C}$-shaped, even if it was not fitted satisfactory by circles or ellipses.

\section{Discussion and Conclusions}

We have presented an original method to study the knee anatomy, which helps to understand the 3D shape of femoral and tibial articulating surfaces more precisely because it is based on multiple sections analysis (compared to single sections/slices [15], [22]) and on acquisition equipment more accurate than X-rays [8, 11, 12], manual measurements on dissected joints [17], [19] and similar to recent methods for kinematic experimental studies [23], [24], [25], [26], [27]. Moreover the numerical description of the tibial shape is an original result of the present study and can be poorly compared with previous qualitative results.

The reported analysis of the tibio-femoral joint suggests a new representation of the bone segments, compatible with some of the previous anatomical models proposed in the literature [8], [10], [21], [11], [13], [14], [15], but providing a more global description in all views and both bone segments. We propose a model of the tibio-femoral joint as made of two rigidly connected ellipsoids with their main axes in 
the AP direction (medial semi-axes $=30$ and $23 \pm 2 \mathrm{~mm}$; lateral semi-axes $=26$ and $20 \pm 4 \mathrm{~mm}$ ), with the ML section circular in the medial side ( $\mathrm{R}=20 \pm 2 \mathrm{~mm})$ and elliptic in the lateral side (semi-axes $=17$ and $9 \pm 2 \mathrm{~mm}$; slope $=160^{\circ}$ ). It articulates on a surface which is a cylinder with its AP axis and circular section on the medial side (conformal to the femoral shape), and a flat wedge on the lateral side, representing the conic or flat tibial eminence and the central part of the tibial plateau. (Figure 2)

This new description of the anatomy of the normal knee suggests that new asymmetric design of artificial knees and non-circular profiles might improve the outcomes of knee reconstruction, reproducing the balance of the natural joint. Moreover an elliptic model of the femoral condyles may explain the apparently different pattern of motion in the first $20-30^{\circ}$ of flexion and the remaining range of flexion as the consequence of a continuous change in the curvature of the articulating surface roughly from the bigger to the smaller ellipse radius.

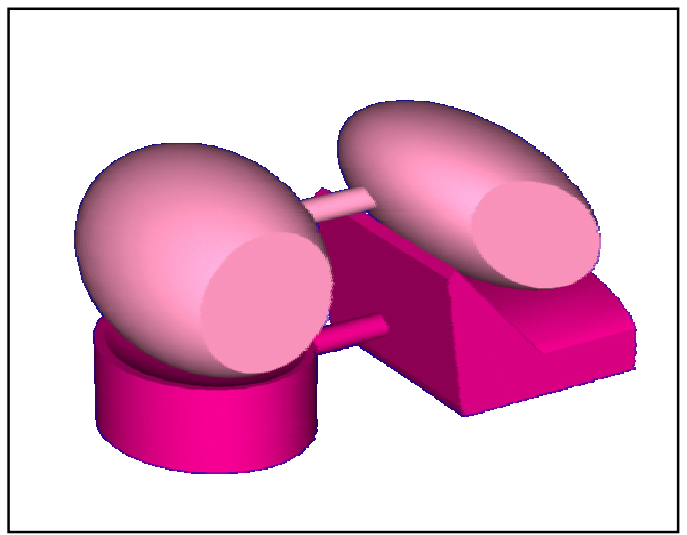

Fig. 2. Anatomical model of the knee joint obtained by computer - dissection (medial compartment on the left, lateral on the right)

\section{References}

1. L. Blankevoort, R. Huiskes : Validation of a three-dimensional model of the knee, J. Biomech., 29(7) (1996) 955-961.

2. H. Kurosawa, P.S. Walker, S. Abe, A. Garg, T. Hunter : Geometry and motion of the knee for implant and orthotic design, J. Biomech., 18(7) (1985) 487-499.

3. D.R. Wilson, J.D. Feikes, J.J. O'Connor : Ligaments and articular contact guide passive knee flexion, J. Biomech., 31 (1998) 1127-1136.

4. W. Weber, E. Weber: Mechanics of the human walking apparatus (Springer-Verlag, Berlin $1992-1^{\text {st }}$ published in german), On the Knee, 4 (1836) 75-92.

5. U. Rehder : Morphometrical studies on the symmetry of the human knee joint: femoral condyles, J. Biomech., 16(5) (1983) 351-361.

6. C.H. Barnett : A comparison of the human knee and avian ankle, J. Anat., 88 (1954) 59-70.

7. T. Röstlund, L. Carlsson, B. Albrektsson, T. Albrektsson: Morphometrical studies of human femoral condyles, J. Biomed. Eng., 11 (1989) 442-448.

8. H. Kurosawa, P.S. Walker, S. Abe, A. Garg, T. Hunter : Geometry and motion of the knee for implant and orthotic design, J. Biomech., 18(7) (1985) 487-499. 
9. J.J. O'Connor, J.W. Goodfellow : The geometry of the knee in the sagittal plane, IMechE., Proc. Instn Engrs, 203 (1989) 223-233.

10. H. Albrecht : XXIII Contribution to the anatomy of the knee joint (translated from German by P. Maquet), Diss. Deutsche Zeits. + Chirurgie III. (1876)

11. J.S. Mensch, C. Harlan, C. Amstutz : Knee morphology as a guide to knee replacement, Clinical Orthopaedics and Related Research, 112 (1975) 231-241.

12. M.J. Erkman, P.S. Walker : A study of knee geometry applied to the design of condylar prostheses, J. Biomech. Eng., 9 (1974) 14-17.

13. T. Röstlund, L. Carlsson, B. Albrektsson, T. Albrektsson: Morphometrical studies of human femoral condyles, J. Biomed. Eng., 11 (1989) 442-448.

14. M. Zoghi, M.S. Hefzy, K.C. Fu, W.T. Jackson: The three-dimensional morphometrical study of the distal human femur, IMechE., Proc. Instn Engrs, 206 (1992) 147-157.

15. V. Pinskerova, H. Iwaki, M. Freeman : The shape and relative movements of the femur and tibia in the unloaded cadaveric knee: a study using MRI as an anatomical tool. In J.N. Insall and W.N. Scott (eds.): Surgery of the knee. $3^{\text {rd }}$ edition, Saunders Inc., Philadelphia USA in press

16. J. Wismans, F. Veldpaus, J. Janssen: A three-dimensional mathematical model of the kneejoint. J. Biomech., 13 (1980) 677-685.

17. V. Pinskerova, P. Maquet, M.A.R. Freeman: Writing on the knee between 1836 and 1917. J Bone and Joint Surg, 82-B(8) (2000) 1100-02.

18. G.S. Langa : Experimental observations and interpretations on the relationship between the morphology and function of the human knee joint. Acta anat., 55 (1963) 16-38.

19. Y. Yoshioka, D.W. Siu, A. Scudamore, T.D.V. Cooke (1989): Tibial anatomy and functional axes. J. Orthop. Res., 7(1), 132-137.

20. Y.Y. Dhaher, L.D. Scott, W.Z. Rymer : The use of basis functions in modelling joint articular surfaces: application to the knee joint. J. Biomech., 33 (2000) 901-907.

21. S. Martelli, V. Pinskerova: The shapes of the tibio-femoral articular surfaces. To appear in J Bone and Joint Surg.

22. A.M. Hollister, S. Jatana, A.K. Singh, W.W. Sullivan, A.G. Lupichuck : The axes of rotation of the knee, Clinical Orthopaedics and Related Research. 290 (1993) 259-268.

23. T.P. Quinn, C.D. Mote : A six degree-of-freedom acoustic transducer for rotation and translation measurements across the knee. J. Biomed. Eng, 112 (1990) 371-378.

24. S. Martelli, S. Zaffagnini, B. Falcioni, M. Marcacci: Intraoperative kinematic protocol for knee joint evaluation, Computer Methods and Programs in Biomedicine, 62, (2000), 77-86.

25. R.J. Minns, W.K. Walsh, J.A. Clarket: Techniques for measuring the static and dynamic properties of the patella. J. Biomed. Eng., 11 (1989) 209-14.

26. H. Fujie, K. Mabuchi, S.L.-Y. Woo, G.A. Livesay, S. Arai, Y. Tsukamoto: The use of robotics technology to study human kinematics: a new methodology. J. Biomed. Eng., 115 (1993) 211-7.

27. L.J. Ruijven, M. Beek, E. Donker, T.M.G.J. van Eijden : The accurancy of joint surface models constructed from data obtained with an electromagnetic tracking device. J. Biomech., 33 (2000) 1023-8. 